\title{
Surface Reconstruction With Multiresolution Discontinuity Analysis
}

\author{
N.F. Law and R. Chung \\ Department of Mechanical and Automation Engineering, \\ The Chinese University of Hong Kong, \\ Shatin, N.T., Hong Kong. \\ email: \{nflaw, rchung $\}$ mae . cuhk. edu.hk
}

\begin{abstract}
The goal of surface reconstruction is to reconstruct a smooth surface while avoiding smoothing out discontinuities. In this paper, a new algorithm for surface reconstruction is proposed which can locate and identify discontinuities while reconstructing a smooth surface from a set of sparse and irregularly spaced depth measurements. This algorithm uses the wavelet transform technique to induce a multiresolution approach for recovering discontinuities. In particular, the wavelet modulus maxima representation is used which allows correlation between wavelet coefficients at different scales. These correlations can be used for feature correspondence across scales. By using this multiresolution information, the estimation of locations of discontinuities is refined. The performance of the algorithm is investigated and compared with a recently published bending moment-based algorithm. It can be seen that our approach can locate and preserve discontinuities while ensuring smoothness in most of the regions.
\end{abstract}

\section{Introduction}

Surface interpolation is a common problem encountered in computer vision, for instance in stereo imaging and visual motion analysis, when a dense depth map of the imaged scene is desirable. It refers to a process in which a piecewise smooth surface is reconstructed from a set of noisy measurements. As identifying and locating discontinuities such as edges and boundaries in the scene are important, we want not only to reconstruct the surface, but also to identify the location of discontinuous points in the reconstruction. This is the goal of the surface reconstruction problem.

As in the case of feature-based stereo imaging, the measurements are obtained through the feature correspondence between the left and right images. It thus gives an irregular sampling pattern and the sampling density could be very sparse. It may also happen that some parts in the image have no measurements as there may be no feature detectable in either the right or the left image. The reconstruction problem is therefore ill-posed in nature. Some additional constraints are needed in order to make the problem well-posed. 
A popular approach to solve this ill-posed problem is by the regularization technique [1], [2]. It restricts the admissible solution to be a smooth function. The problem can be formulated as minimizing an error function defined as,

$$
E=\sum_{k=1}^{K}\left(z_{k}-f\left(x_{k}, y_{k}\right)\right)^{2}+\lambda S(f)
$$

where $K$ is the total number of measurements available, the first term is the data constraint, i.e., the residual error in surface fitting to the measurements, the second term $S(f)$ is the smoothness requirement placed on $f$, and $\lambda$ is a regularization constant which controls the tradeoff between the data constraint and the smoothness constraint. One popular choice for the functional $S(f)$ is the following quadratic form,

$$
S(f)=\iint\left[\left(D_{x}^{2} f\right)^{2}+2\left(D_{x} D_{y} f\right)^{2}+\left(D_{y}^{2} f\right)^{2}\right] d x d y
$$

where $D_{x}$ and $D_{y}$ are the differential operators with respect to $x$ and $y$ respectively.

As the smoothness constraint is applied globally to the entire scene, discontinuities present serious difficulties to the above formulation. Most often, discontinuities are blurred by minimizing $E$ without a proper choice of $\lambda$. Different methods have been proposed to relax the global smoothness constraint in various ways so as to preserve visual discontinuities. The basic philosophy is to apply different degrees of smoothness to different parts of the image as in [2], [3], [4]. However, the discontinuities are analyzed using single resolution methods. As pointed out in [5], multiresolution analysis performs better than single resolution in discriminating between noise and desired features.

Another difficulty encountered in this minimization problem is the slow convergence rate. It becomes particularly serious when the size of the problem is large. A way to improve the convergence rate is to employ multigrid processing which basically uses the multiresolution concept in improving the convergence rate [2].

Wavelet transform provides a multiresolution picture of an object and thus enables multigrid processing. There are many different kinds of scheme for wavelet transform. One popular choice is the orthogonal wavelet transform as in [6]. An orthogonal wavelet is used as a preconditioning transform, i.e., the wavelet transform is applied to diagonalize the linear equation system. However, the discontinuities are found separately using the bending moment method rather than using the multiresolution property inherent to the wavelet transform [8]. In this paper, the scheme that we choose is the wavelet modulus maxima representation used by Mallat and Zhong [7]. This representation has a property that there exists strong correlation between the wavelet coefficients across scales. Noise would usually show up in the first few wavelet bands with its effect decreases drastically in the higher wavelet bands. In contrast strong edges will have their effects showing across all wavelet bands. Hence, by tracking the effects across wavelet 
bands, we can establish whether the response is due to noise or not, and how strong the edge is [8].

We propose to make use of this property to establish correlations between the wavelet coefficients across different scales. These correlations can then enhance the estimations of the wavelet coefficients at different scales and enable the discontinuities to be estimated robustly in a multiresolution manner. Experimental results show that our approach can locate and preserve discontinuities and reconstruct the surface with good quality even when the measurements are sparse.

In summary, our proposed algorithm allows surface reconstruction and discontinuities detection to be carried out simultaneously and under the same wavelet framework. Besides, the multiresolution approach inherent in the wavelet formulation allows a more robust performance in discontinuity detection.

\section{Multiresolution Approach in the Wavelet Framework}

Discretizing Eqns (1) and (2) gives the following expression for the surface reconstruction error,

$$
\begin{aligned}
E= & \sum_{k=1}^{K}\left(z_{k}-f\left(m_{k}, n_{k}\right)\right)^{2}+\sum_{i, j} \lambda(i, j) A^{2}(i, j) \\
& \sum_{i, j} \lambda(i, j) B^{2}(i, j)+2 \sum_{i, j} \lambda(i, j) C^{2}(i, j)
\end{aligned}
$$

where

$$
\begin{aligned}
A(i, j)= & f(i, j+1)-2 f(i, j)+f(i, j-1) \\
B(i, j)= & f(i+1, j)-2 f(i, j)+f(i-1, j) \\
C(i, j)= & f(i+1, j+1)-f(i+1, j) \\
& -f(i, j+1)+f(i, j)
\end{aligned}
$$

$A, B$ and $C$ are the discrete approximations to the differential operations $D_{x}^{2}$, $D_{y}^{2}$ and $D_{x} D_{y}$ respectively. The image up to a particular resolution can be represented using the quadratic spline wavelets as follows [5],

$$
\begin{aligned}
f_{a}(m, n)= & \sum_{i} \sum_{j} h_{a}(m, i) s_{a}(i, j) h_{a}(n, j) \\
& +\sum_{i} \sum_{j} l_{a}(m, i) w^{1}{ }_{a}(i, j) k_{a}(n, j) \\
& +\sum_{i} \sum_{j} k_{a}(m, i) w^{2}{ }_{a}(i, j) l_{a}(n, j)
\end{aligned}
$$

or in matrix-vector notation as,

$$
\mathbf{f}_{a}=\mathbf{H}_{a} \mathbf{S}_{a} \mathbf{H}_{a}{ }^{T}+\mathbf{L}_{a} \mathbf{W}^{1}{ }_{a} \mathbf{K}_{a}{ }^{T}+\mathbf{K}_{a} \mathbf{W}^{2}{ }_{a} \mathbf{L}_{a}{ }^{T}
$$


where $a$ is the resolution level, $f_{a}$ is the image which consists of information only up to $(a-1)^{\text {th }}$ level, $s_{a}$ is the lowpass version of $f$ at level $a, w^{1}{ }_{a}$ and $w^{2}{ }_{a}$ are the wavelet coefficients at horizontal and vertical directions respectively, $h_{a}, l_{a}$ and $k_{a}$ are the reconstruction filters for $s_{a}, w^{1}{ }_{a}$ and $w^{2}{ }_{a}$ and are defined in [8].

Our approach is first to reconstruct an overly smooth solution and then refine the discontinuities iteratively in the overly smooth surface. In our formulation, the first smooth surface is the lowpass portion of the image at a particular resolution. The smooth surface estimation could be done by assuming $w^{1}{ }_{a}$ and $w^{2}{ }_{a}$ to be zero, and then choose the lowpass coefficient $s_{a}$ so that the resultant $f_{a}$ minimizes $E$. The minimization is done by the steepest descent search for simplicity. The gradient of $E$ with respect to $s_{a}(m, n)$ is given by,

$$
\begin{aligned}
\frac{\partial E}{\partial s_{a}}= & -2 \sum_{k=1}^{K}\left(z_{k}-f\left(m_{k}, n_{k}\right)\right) D\left(m_{k}, n_{k}, m, n\right) \\
& +2 \sum_{i, j} \lambda(i, j) A(i, j) A^{\prime}(i, j) \\
& +2 \sum_{i, j} \lambda(i, j) B(i, j) B^{\prime}(i, j) \\
& +4 \sum_{i, j} \lambda(i, j) C(i, j) C^{\prime}(i, j)
\end{aligned}
$$

where

$$
\begin{aligned}
A^{\prime}(i, j)= & D(i, j+1, m, n)-2 D(i, j, m, n) \\
& +D(i, j-1, m, n) \\
B^{\prime}(i, j)= & D(i+1, j, m, n)-2 D(i, j, m, n) \\
& +D(i-1, j, m, n) \\
C^{\prime}(i, j)= & D(i+1, j+1, m, n)-D(i+1, j, m, n) \\
& -D(i, j+1, m, n)+D(i, j, m, n) \\
D(i, j)= & h_{a}(i, k) h_{a}(j, l)
\end{aligned}
$$

The derivative of $E$ with respect to $w^{1}{ }_{a}$ and $w^{2}{ }_{a}$ can be obtained accordingly. The actual implementation is done in the Fourier domain for computational efficiency.

After having a set of coefficients for $s_{a}$, we then estimate the bandpass coefficients $w^{1}{ }_{a}$ and $w^{2}{ }_{a}$ so that the resultant surface with discontinuities minimizes $E$ further. In this way, the bandpass information is progressively added into the reconstruction process. The derivative of $E$ with respect to $w^{1}{ }_{a}$ and $w^{2}{ }_{a}$ can be obtained from Eq 4 in the same way as $\frac{\partial E}{\partial s_{a}}$ in Eq 7 .

At the same time when the bandpass information is added to the reconstructed surface, we could track the wavelet modulus maxima positions across scales to find out whether the modulus maxima positions are due to noise (incorrect estimation) or due to discontinuities. Thus the estimations are improved by incorporating the multiresolution constraints inherent in our formulation. We 
could also impose the constraint that the modulus maxima cannot be created as one moves from fine to coarse scales. In this way, we could estimate where the discontinuities are robustly in the multiresolution framework.

The individual wavelet transform modulus maxima represent discontinuities. However, in two or higher dimensions, they are usually not independent features; they refer to points or extended boundaries and belong to certain lines or curves. Hence, we could link the individual wavelet modulus maxima to form contours and use these contours as primitives in feature correspondence across different scales.

In forming the wavelet modulus maxima contours, it is necessary to discard weak and short contours to facilitate the feature correspondence process. The boundaries of important coherent structures often generate long contour curves whereas contours resulting from noise are short. We thus remove any contour curve whose length is smaller than a given length threshold. Also, contour that has a low average amplitude corresponds to small variation in the image which is ignored. The length threshold and the amplitude threshold are set to be $30 \%$ of their respective mean values of all contours in an image. We keep only those contours that satisfy both the length threshold and the amplitude threshold. Thus, the thresholds for both length and amplitude are set automatically for every test image.

The criteria used for features correspondence are position, sign and amplitude of the contours. The determining factor is the sign. If the sign between two modulus maxima contours at two scales are not the same, then they do not match with each other. If the sign is correct, check the position. The positional tolerance which is determined by the width of the wavelet reproducing kernels is used to decide whether the contours are close or not. If the contours are close together, check the amplitude to see if they are similar. It should be noted that the matching between the fine scale and the coarse scale can only be a many-toone mapping, but not a one-to-many mapping. Since we match contours instead of individual pixels, we can either keep the contour if we find a match or remove the whole contour if no match is found.

If we consider the resolution level to be three, our algorithm can be summarized as follows,

1. Obtain a lowpass function $s_{3}$ by assuming no discontinuities;

2. Based on $s_{3}$, estimate $w^{1}{ }_{3}$ and $w^{2}{ }_{3}$ by using the gradient search to minimize the error defined in $\mathrm{Eq} 4$. A rough estimate of the discontinuities points can be obtained based on $f_{3}$ and $\lambda$ is updated accordingly.

3. Based on the above estimated $f_{3}$ (ie., $w^{1}{ }_{3}, w^{2}{ }_{3}$ and $s_{3}$ and hence $s_{2}$ ), estimate $w^{1}{ }_{2}$ and $w^{2}{ }_{2}$. Then extract the modulus maxima of both pairs $w^{1}{ }_{2}, w^{1}{ }_{3}$ and $w^{2}{ }_{2}, w^{2}{ }_{3}$ and apply the feature correspondence to shape $w^{1}{ }_{2}, w^{1}{ }_{3}$ and $w^{2}{ }_{2}$, $w^{2}{ }_{3}$. Through the above procedure, some unlikely or unmatched contours are clean up and smoothed out and we could estimate where the discontinuous points are and update $\lambda$ accordingly.

4. Based on the above estimated $f_{2}$, estimate $w^{1}{ }_{1}$ and $w^{2}{ }_{1}$. Then apply feature correspondence to shape the wavelet coefficients at different scales and up- 
date $\lambda$ accordingly. Repeat this step until the estimated $f$ does not change much.

A popular choice for $\lambda(i, j)$ is a binary set, ie., $\{0,1\}$. If a particular pixel $(i, j)$ is known to be a discontinuous point, then the smoothness constraint is turned off by setting $\lambda(i, j)$ to zero, otherwise, the smoothness constraint is enforced by setting $\lambda(i, j)$ to one. We also adopt this approach except that $\lambda(i, j)$ in our case is determined by the wavelet maxima contours. At where the wavelet maxima contours are found, $\lambda$ is set to zero and at where the wavelet maxima contours are not found, $\lambda$ is set to one. In this way, we ensure that discontinuities are connected, they are not isolated features which could be due to noise or incorrect estimation.

\section{Experimental Results}

We provide some experimental results to illustrate the edge-detection and edgepreserving performance of our surface reconstruction algorithm. The proposed algorithm is applied to various data sets including both synthetic and real images. Its performance as a surface reconstruction algorithm was demonstrated on three images shown in Fig 1. For comparison purpose, a recently published bending moment-based algorithm [6] is also applied to the same images.

Fig 2 shows synthetic range measurements where zero-valued means no sample data and the intensity denotes the range. A sampling density of $10 \%$ of the range image is used. Fig 3 shows the discontinuities found and the reconstructed surface using our algorithm with three resolution levels and 50 iterations in each level. Fig 4 shows the discontinuities found and the reconstructed surface using the bending moment-based algorithm. A number of edge thresholds have been tried and the best discontinuity map is obtained by setting the threshold to be 150. It can be seen that the bending moment-based algorithm fails to reconstruct the three-level surface and the discontinuities detected are incorrect. Despite the sparse data available over a surface with a lot of discontinuities, our algorithm gives a good reconstruction.

In the bending moment-based algorithm, the discontinuities are detected using the bending moment method rather than using the multiresolution framework inherent to the wavelet transform. Moreover the off-diagonal terms of the preconditioned equation system are all approximated to be zero. The effect of this diagonalization depends on the regularization constant, the sampling density and the reconstruction level chosen. Thus, the diagonal assumption may not be valid at all times. In contrast, our algorithm uses the multiresolution information in constructing the discontinuity map which thus enhance the estimation of the wavelet coefficients and give a good solution.

In the second example, a checkerbox surface of size $128 \times 128$ is interpolated. The sampling density is also $10 \%$ and the sampled image is shown in Fig 5 . Fig 6 shows the discontinuities found and the reconstructed surface using our algorithm with three resolution levels and 50 iterations in each level. Fig 7 shows 


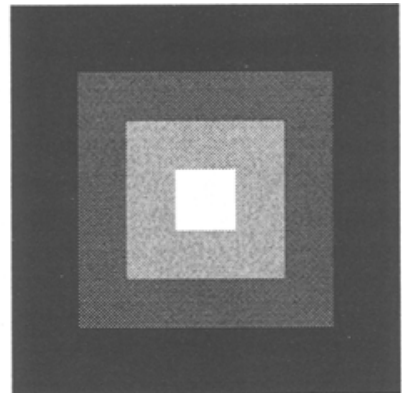

(a)

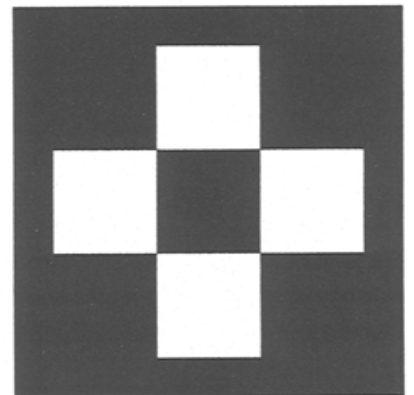

(b)

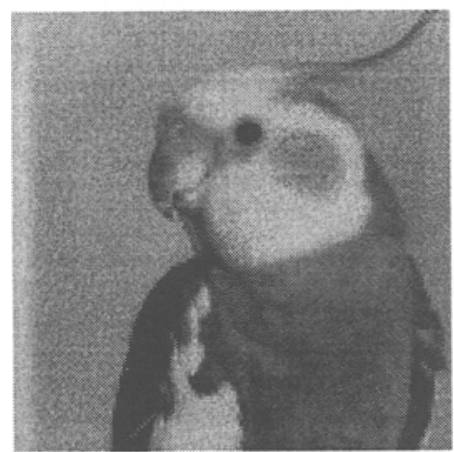

(c)

Fig. 1. The images used to test the surface reconstruction algorithm, (a) the wedding cake range image; (b) a checkerboard image; and (c) a bird image 


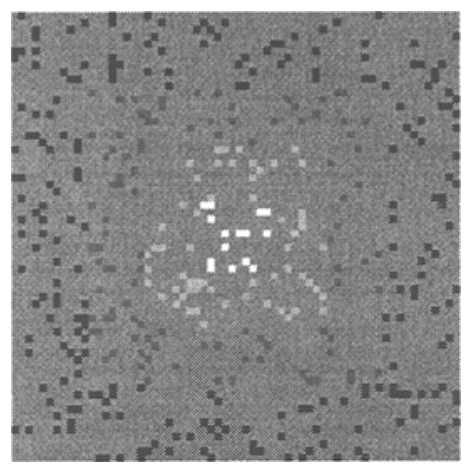

Fig. 2. $10 \%$ sampling density of the wedding cake image. Dots indicate the sampled data, and the brightness on them indicate the depth. The brighter the point is, the closer to the viewer it is.

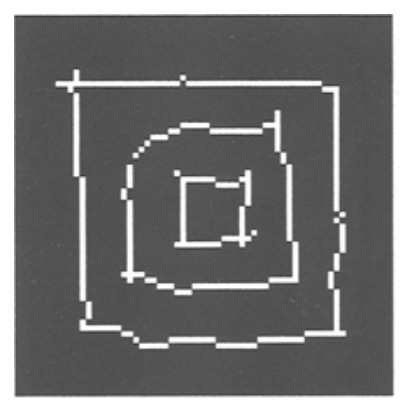

(a)

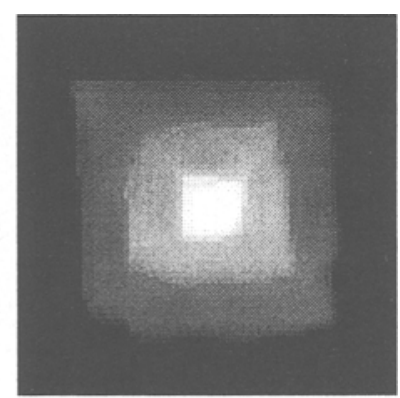

(b)

Fig. 3. The results obtained with the proposed algorithm for the $10 \%$ wedding cake range data; (a) the discontinuities found and (b) the reconstructed surface. 


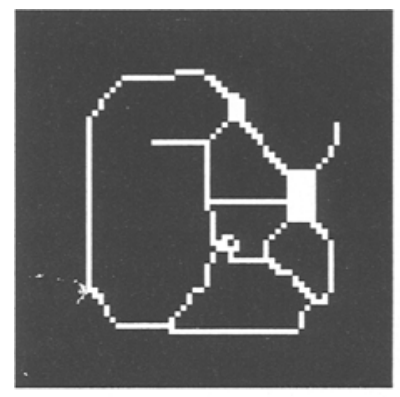

(a)

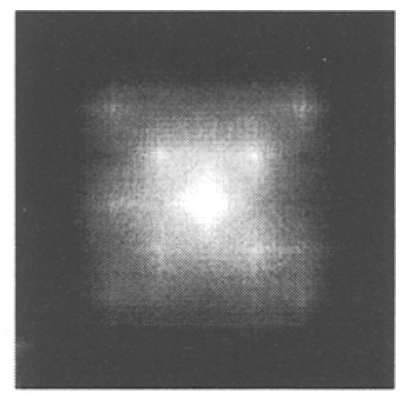

(b)

Fig. 4. The results obtained with the bending moment-based algorithm for the wedding cake range data; (a) the discontinuities found and (b) the reconstructed surface. The resolution level is set to be three and the edge threshold is 150 .

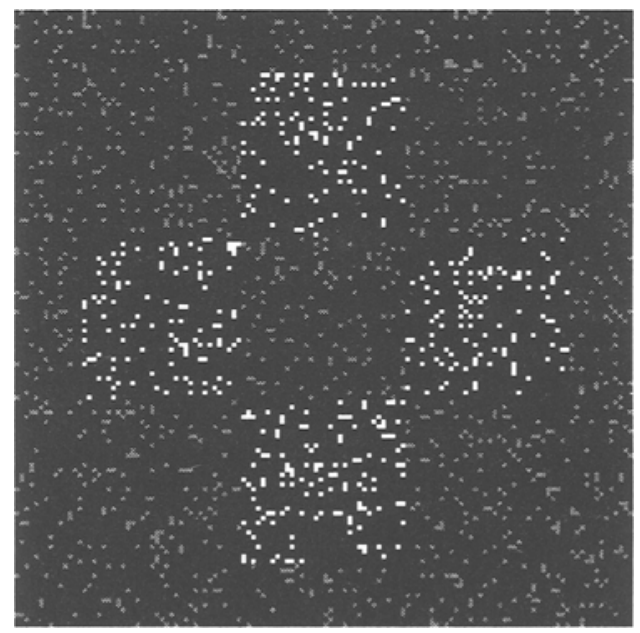

Fig. 5. A $10 \%$ sampling density of the checkerboard image. Dots indicate the sampled data, and the brightness on them indicate the depth. The brighter the point is, the closer to the viewer it is. 


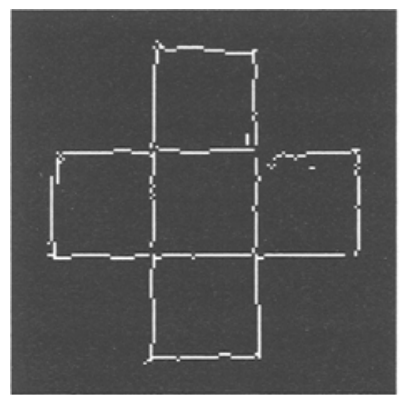

(a)

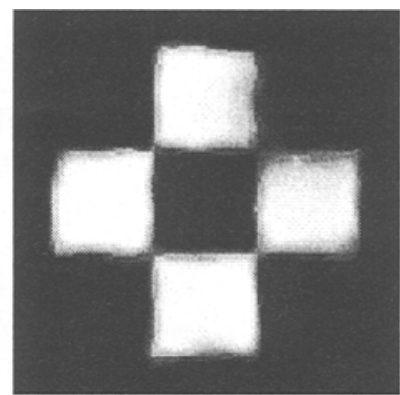

(b)

Fig. 6. The results obtained with the proposed algorithm for the $10 \%$ checkerboard image; (a) the discontinuities found and (b) the reconstructed surface.

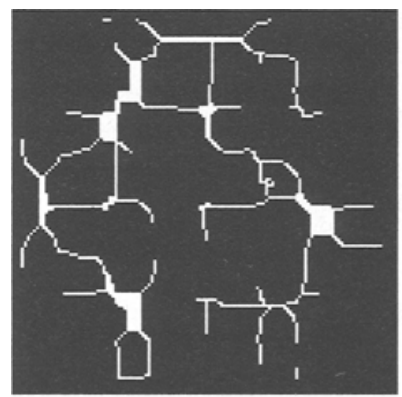

(a)

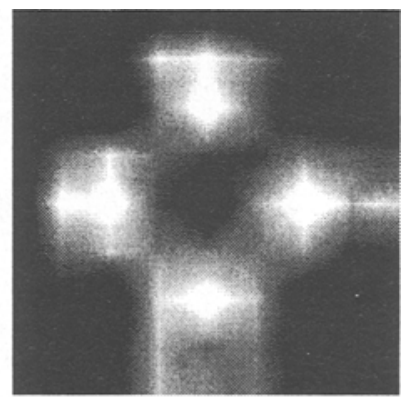

(b)

Fig. 7. The results obtained with the bending moment-based algorithm for the checkerboard image; (a) the discontinuities found and (b) the reconstructed surface. The resolution level is set to be four and the edge threshold is 150 . 
the edges found and the reconstructed surface using the bending moment-based algorithm.

We have experienced some difficulties in the bending moment-based algorithm in this example. When the resolution level is chosen to be four, the results are not good and the discontinuities detected using bending moment are messy. But if the resolution level is set to three, the reconstructed surface looks better except that some artifacts appear in the reconstructed surface as evidenced in Fig 8. This happens because the diagonalization assumption which depends on resolution level and the sampling density is not valid. However, our algorithm can still give a good reconstruction.

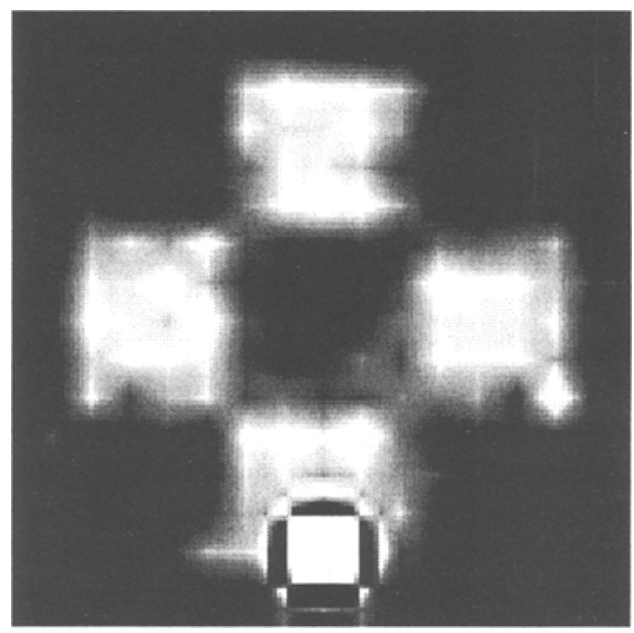

Fig. 8. The reconstructed surface using the bending moment-based approach. The resolution level is set to be three, and the number of iterations is two. Artifacts are obvious in the reconstructed surface.

In the above two examples, we can see that the bending moment-based algorithm do not give a good reconstruction when the sampling density is only $10 \%$. The discontinuity map which is obtained in a single resolution framework is also not as good as the multiresolution discontinuity detection. We have also tried on some higher sampling density case to see how the new algorithm compares with the bending moment-based algorithm. The results shown here are about the image of a bird. The sampling density is $30 \%$ in this example. Fig 9 shows the reconstructed surfaces using our algorithm and the bending momentbased algorithm. The reconstruction of the bending moment-based algorithm is improved. However, our algorithm again provides a better reconstruction. 


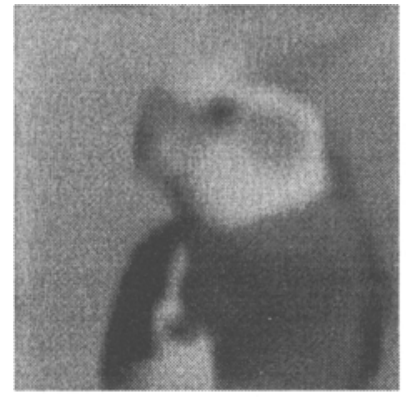

(a)

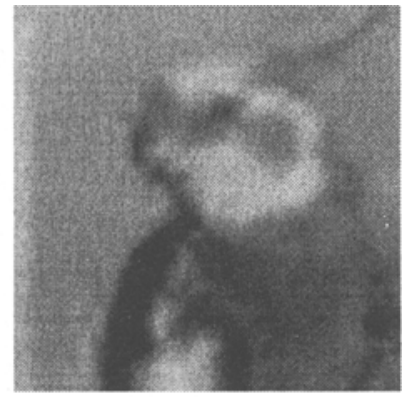

(b)

Fig. 9. The reconstructed surfaces obtained by (a) our proposed algorithm and (b) bending moment-based algorithm for a $30 \%$ sampling density of the bird image.

\section{Conclusion}

Global smoothness constraints intrinsic to standard regularization are inadequate near discontinuities. Since discontinuities play a vital role in inverse visual problems, some ways are needed to detect their locations so as to avoid smoothing over the discontinuous points. Typical ways to analyze discontinuities are, however, single resolution method in nature. We have presented in this paper a new surface reconstruction method which combines the multiresolution discontinuity tracking and the wavelet transform techniques to reconstruct from a sparse set of measurement a piecewise smooth surface that preserve discontinuities.

The new algorithm uses the wavelet modulus maxima representation [7]. Because of the multiresolution property of the representation and the existence of correlations between wavelet coefficients at different scales, feature correspondence across scales is possible. This improves the estimation of discontinuities and enhance the performance of the surface reconstruction algorithm.

The new algorithm starts with an overly smoothed surface. Discontinuities information which are characterized by the wavelet modulus maxima are added to the reconstructed surface progressively. Thus the discontinuity map undergoes refinement during surface reconstruction.

The new algorithm has been tested on a number of synthetic and real data sets. Simulation results show that the approach can preserve the discontinuities while ensuring smoothness in most regions. Comparison of the proposed algorithm with a recently published algorithm [6] has also been presented. From the experimental results, the performance of our algorithm has been shown to be superior in the surface reconstruction problem. 


\section{Acknowledgments}

N.F. Law thanks the Chinese University of Hong Kong for the support under its Postdoctoral Fellowship on this research. The research was also partially supported by RGC of Hong Kong under the 1994-1995 Earmarked Grant.

\section{References}

1. Tomaso Poggio, Vincent Torre and Christof Koch, "Computational vision and regularization theory", Nature, Vol. 317, Sept 1985, pp 314-319.

2. D. Terzopoulos, "Regularization of Inverse Visual Problems Involving Discontinuities", IEEE Trans on Pattern Analysis and Machine Intelligence, Vol. 8, No. 4, July, 1986 , pp $413-423$.

3. D. Terzopoulos, "The Computation of Visible Surface Representations", IEEE Trans on Pattern Analysis and Machine Intelligence, Vol. 10, No. 4, July, 1988, pp 417-437.

4. M. Gokmen and C.C. Li, "Edge Detection and Surface Reconstruction Using Refined Regularization", IEEE Trans on Pattern Analysis and Machine Intelligence, Vol. 15, No. 5, May, 1993, pp 492-499.

5. A.W.C. Liew, "Multiscale Wavelet Analysis of Edges: Issues of Uniqueness and Reconstruction", $\mathrm{PhD}$ thesis, University of Tasmania, Australia, 1996.

6. Alex P Pentland, "Interpolation Using Wavelet Bases", IEEE Transactions on Pattern Analysis and Machine Intelligence, Vol. 16, No. 4, April, 1994, pp 410 - 414.

7. Stephane Mallat and Sifen Zhong, "Characterization of Signals from Multiscale Edges", IEEE Transactions on Pattern Analysis and Machine Intelligence, Vol. 14, No. 7 , July 1992 , pp $710-732$.

8. N.F. Law and R. Chung, "Surface Reconstruction With Multiresolution Discontinuity Analysis", Technical Report, CUHK-MAE-98-04, The Chinese University of Hong Kong, 1998. 